\title{
International News
}

RCOG World Congress - 2019

Date: 17-19 June 2019

Venue: London, UK

Website: www.rcog.org.uk

ICGO 2019 : 21st International Conference on Gynecology and Obstetrics

Date: September 25 - 26, 2019

Venue: London, United Kingdom

Website: waset.org/conference/2019/09/london/

ICGO

8th IWA-ASPIRE Conference \& Exhibition 2019

Date: 31.10 .2019 to 3.11.2019

Venue: Hong Kong Convention and Exhibition Centre Website: www.iwaaspire2019.org

\section{$2^{\text {th }}$ AOCOG Congress}

Date: 11-16 November 2019

Venue: Manila, Philippines

Website: www.aocog2019.com

\section{3rd AICC RCOG Conference}

Date : 05/09/2019 - 07/09/2019

Venue : ITC Royal Bengal, Kolkatta

Contact Person : Dr Bhaskar Pal

Mobile : 9831298236

Email : palbas@hotmail.com

\section{FOGSI Conference on High Risk Pregnancy}

Date : 24/08/2019 - 25/08/2019

Venue : Ahmedabad

Contact Person : Dr Haresh Doshi

Mobile : 9825060125

Email : doshiharesh@hotmail.com

Gynecology and Infertility Chronicles

Date : 14/09/2019 - 15/09/2019

Venue : FOGSI Office, Mumbai

Contact Person : Dr Jaydeep Tank

Mobile : 9820106354

Email : drjaydeeptank@gmail.com
Breaking Silos across Adolescence to Menopause

Date : 21/09/2019 - 22/09/2019

Venue : Pravasi Bhartiya Kendra, New Delhi

Contact Person : Dr Sudha Prasad

Mobile : 9968604341

Email : drsprasad@yahoo.com

The $9^{\text {th }}$ Congress of the Asia Pacific Initiative on Reproduction (ASPIRE 2019)

Date: 2 - 5 May 2019

Venue: Hong Kong Convention and Exhibition Centre.

Website: www.aspire2019.com

$27^{\text {th }}$ World Congress on Controversies in Obstetrics, Gynecology and Infertility

Date: November 21-23, 2019.

Venue: Paris, France,

Website. http://cogi-congress.org

63rd All India Congress of Obstetrics and Gynaecology (AICOG)

Jan 29 - Feb 02, 2020

Lucknow, Uttar Pradesh

Website: www.aicog2020lucknow.in

$21^{\text {st }}$ Congress of the Federation of Asia and Oceania Perinatal Societies

Date: $28^{\text {th }}$ November $-1^{\text {st }}$ December 2020

Venue: Tokyo International Forum

Website: www.faops2020.com

FIGO's XXIII World Congress

Venue: Sydney, Australia,

Date: 24-29 October 2021 